\title{
The significance of GATA3 expression in breast cancer: a 10-year follow-up study.
}

\author{
Vincenzo Ciocca \\ Thomas Jefferson University \\ Constantine Daskalakis \\ Thomas Jefferson University \\ Robin M. Ciocca \\ Fox Chase Cancer Center \\ Alejandra Ruiz-Orrico \\ Thomas Jefferson University \\ Juan P. Palazzo

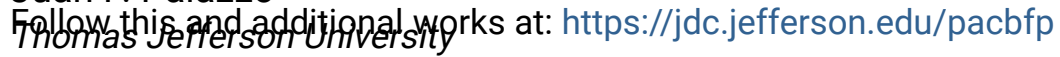 \\ Part of the Medical Cell Biology Commons \\ Let us know how access to this document benefits you
}

\section{Recommended Citation}

Ciocca, Vincenzo; Daskalakis, Constantine; Ciocca, Robin M.; Ruiz-Orrico, Alejandra; and Palazzo, Juan P., "The significance of GATA3 expression in breast cancer: a 10-year follow-up study." (2009). Department of Pathology, Anatomy, and Cell Biology Faculty Papers. Paper 21. https://jdc.jefferson.edu/pacbfp/21

This Article is brought to you for free and open access by the Jefferson Digital Commons. The Jefferson Digital Commons is a service of Thomas Jefferson University's Center for Teaching and Learning (CTL). The Commons is a showcase for Jefferson books and journals, peer-reviewed scholarly publications, unique historical collections from the University archives, and teaching tools. The Jefferson Digital Commons allows researchers and interested readers anywhere in the world to learn about and keep up to date with Jefferson scholarship. This article has been accepted for inclusion in Department of Pathology, Anatomy, and Cell Biology Faculty Papers by an authorized administrator of the Jefferson Digital Commons. For more information, please contact: JeffersonDigitalCommons@jefferson.edu. 
As submitted to Human Pathology and later published as:

"The significance of GATA3 expression in breast cancer: a 10-year follow-up study"

In Human Pathology

Volume 40, Issue 4, April 2009, Pages 489-495

DOI: 10.1016/j.humpath.2008.09.010

\section{THE SIGNIFICANCE OF GATA3 EXPRESSION IN BREAST CANCER: A 10 YEAR FOLLOW-UP STUDY}

Vincenzo Ciocca*, Constantine Daskalakis†, Robin M. Ciocca $\ddagger$, Alejandra Ruiz-Orrico*, and Juan P. Palazzo*.

From the Departments of *Pathology and ${ }^{\dagger}$ Biostatistics, Thomas Jefferson University

Hospital, 132 South $10^{\text {th }}$ Street, Philadelphia, PA 19107, and the Department of

\$Surgical Oncology, Fox Chase Cancer Center, 333 Cottman Avenue, Philadelphia, PA, 19111.

Running Title: Breast cancer and GATA3

Keywords: GATA3; Breast cancer; Tamoxifen 


\title{
Address all correspondence and reprint requests to:
}

Juan P. Palazzo MD

Thomas Jefferson University Hospital

132 S. $10^{\text {th }}$ Street

285 Main Building

Philadelphia, PA 19107

215-955-6352 (telephone)

215-923-1969 (fax)

Juan.Palazzo@jefferson.edu

\begin{abstract}
Aims. GATA3 is a transcription factor closely associated with estrogen receptor alpha (ER) in breast carcinoma, with a potential prognostic utility. This study investigated the immunohistochemical expression of GATA3 in ER-positive and ER-negative breast carcinomas.
\end{abstract}

Methods and Results. One hundred sixty six cases of invasive breast carcinomas with 10-year follow-up information were analyzed. Positive GATA3 and ER cases were defined as greater than $20 \%$ of cells staining. Time to cancer recurrence and time to death were analyzed with survival methods. Of 166 patients, 40 were ER-negative and 121 ERpositive. Thirty eight (23\%) recurrences and $51(31 \%)$ deaths were observed. In final multivariable analyses, GATA3-positive tumors had about two-thirds the recurrence risk of GATA3-negative tumors (hazard ratio, $\mathrm{HR}=0.65, \mathrm{p}=0.395$ ) and comparable mortality risk $(H R=0.86, p=0.730)$. In pre-specified subgroup analyses, the protective effect of GATA3 expression was most pronounced among ER-positive patients who received tamoxifen $(\mathrm{HR}=0.57$ for recurrence and 0.68 for death) 
Conclusion. We found no statistically significant differences in recurrence or survival rates between GATA3-positive and GATA3-negative tumors. However, there was a suggestion of a modest-to-strong protective effect of GATA3 expression among ERpositive patients receiving hormone therapy.

\section{Introduction}

GATA3 is one of six members of a family of transcription factors that bind the consensus motif A/TGATAA/G and which regulates critical steps of differentiation during embryonic development [1]. GATA3 is located at chromosome 10p15 [2]. GATA 1, 2 and 3 are critically involved in myeloerythropoiesis whereas GATA 4, 5 and 6 are implicated in cardiac and intestinal development [3]. The binding element comprises two C4 zinc finger motifs shared with the steroid hormone receptor super family, and is highly conserved among different vertebrate and invertebrate species [1]. GATA3 regulates lineage determination of many cell types. These include neurons of the sympathetic nervous system, $\mathrm{T}$ helper cells, the inner root sheath of hair follicles, fat cells, nephritic ducts of the kidney, and the ear cochleae $[4,5]$. Fetal mice cannot survive to term without GATA3 [6]. In humans, mutations in one of the GATA3 genes lead to thyroid and kidney dysfunctions, and hearing abnormalities [7]. Two studies showed that GATA3 is necessary for mammary gland morphogenesis, luminal-cell differentiation and maintenance of luminal cell differentiation in adult mice $[8,9]$. GATA3 expression occurs early in embryonic development, but only in the luminal epithelial cells, not it myoepithelial cells. 
GATA3 is a gene regulatory factor that guides immature breast cells on their developmental path to form luminal epithelial cells. Moreover, GATA3 is linked to two other gene regulatory factors, namely FoxA1 and estrogen receptor alpha [10]. GATA3 is normally expressed at high levels, in association with ER, in the luminal epithelial cells. Interestingly, the loss of GATA3 expression results in decreased ER expression. GATA3 and ER are involved in a positive cross-regulatory loop, where each one of these factors is required for the transcription of the other gene. Furthermore, GATA3 and ER autoregulate their own expression. These mechanisms could explain the high correlation of GATA3 and ER expression in luminal A breast tumors [10]. Therefore, by regulation of ER expression, GATA3 is crucial for estradiol-stimulated progression of ER-positive breast cancer. Luminal A breast cancers show the highest expression of ER and GATA3. Luminal B tumors show significantly decreased ER and GATA3 expression while basallike cancers show the lowest expression of ER and GATA3 [11, 12].

Expression of ER is currently the best approach to predicting whether or not breast carcinoma will respond to hormonal therapy. However, approximately $30 \%$ of ER-positive breast cancers do not respond to hormonal therapy. In breast cancer, it has been shown that higher expression of GATA3 predicts a better prognosis and GATA3 expression has been shown to be a predictor of hormonal response [13-15]. The goal of this study is to correlate GATA3 expression with recurrence and survival in ER-positive breast cancer patients with 10 years of follow-up. 


\section{Materials \& Methods}

Formalin fixed paraffin-embedded tissues of consecutive breast carcinomas were obtained from the files of the Department of Pathology of Thomas Jefferson University. All breast tissue was formalin fixed appropriately following a protocol for breast samples that was established in our Institution to avoid false negative or positive staining. All cases had been treated with surgery (most by lumpectomy alone and $25 \%$ with mastectomy); the majority of these patients had also received radiotherapy, and some patients had received hormonal therapy and/or chemotherapy. The cases were stained with GATA3 monoclonal antibody (Santa Cruz Biotechnology, 1:250 dilution) with microwave antigen retrieval pretreatment in citrate buffer as previously described [14]. Positive and negative controls were used in each run. The study was approved by the Thomas Jefferson University Internal Review Board.

We analyzed time to recurrence and time to death via survival methods, specifically the Kaplan-Meier approach and the logrank test, as well as Cox proportional hazards regression. The final multivariable model included five markers (GATA3, ER, PR, Ki67, and p53) irrespective of their statistical significance; other variables were retained if their $\mathrm{p}$-value was less than 0.1 . For the main analyses, the expression of all five markers was dichotomized a priori as nuclear staining in $0-20 \%$ versus $21-100 \%$ of the cells. We also explored the significance between lower and higher cut points (greater than or less than 20\%) for GATA3 as well as its expression as a continuous predictor. Additional analyses explored different cut points, as well as the use of the markers' expression as a continuous predictor. GATA3 immunohistochemical scoring was performed blinded by two pathologists (V.C. and J.P.P.). GATA3 nuclear staining of any 
intensity was considered positive when present in $20 \%$ or more of the tumor cells. Statistical analyses were carried out in SAS 8.2 (SAS Institute Inc., Cary, NC).

\section{Results}

Information from 188 patients was reviewed, and the final analysis sample consisted of 166 patients with available data for key variables (including follow-up dates and GATA3 expression). Clinical follow-up ranged between 4 and 133 months (median = 115 months). The mean age at diagnosis was 62 years (range, 27 to 89 years) so that all age groups were represented in the study.

Tables 1 and 2 present details of the study patients' tumor and treatment characteristics, respectively. The majority of tumors were invasive ductal carcinomas (IDC) and tumor size ranged from 0.4 to $10 \mathrm{~cm}$. The vast majority of patients presented with stage 1 or stage 2 (AJCC) carcinomas. Of the $125 \mathrm{~T} 1$ invasive carcinomas, 68 (54\%) were GATA3-positive. Of the 31 T2 invasive carcinomas, 23 (74\%) were GATA3-positive. Of the seven T3 tumors, 6 were invasive ductal carcinomas and 1 was invasive lobular carcinoma. Of these seven T3 tumors, one tumor (invasive ductal carcinoma) was ER-positive and GATA3-positive. The remaining tumors were all ERnegative and GATA3-negative.

Most of the tumors stained positive for both ER and GATA3 (Figures 1-6). There was a very strong correlation between GATA3 and ER expression. Of the 93 tumors that were ER-positive/GATA3-positive, expression tended to be very high (i.e., 80-90\%) for both markers. Of the ER-negative tumors, all but 2 were also GATA3-negative, while of the 121 ER-positive patients, 93 (77\%) were also GATA3 positive. There were 12 cases 
of invasive lobular carcinoma. Eleven of the twelve invasive lobular carcinomas were ER-positive. Of these 11 tumors, 6 were GATA3-positive and 4 were GATA3-negative (one paraffin block had insufficient tissue for GATA3 staining). One invasive lobular carcinoma was ER-negative and GATA3-negative. The percentage of ER-positive, GATA3-positive tumors is slightly lower for invasive lobular than invasive ductal carcinomas, $60 \%$ and $77 \%$, respectively.

\subsection{GATA3 and Recurrence}

A total of $38(23 \%)$ cancer recurrences were observed, including 13 local, 1 regional, and 24 metastatic. Among the 38 patients, time from surgery to recurrence ranged from 4.4 to 94.1 months (median $=19.5$ months). Of the 121 ER-positive tumors, 93 were GATA3-positive and 28 were GATA3-negative. Of the 45 ER-negative tumors, 43 were GATA3-negative and 2 were GATA3-positive. Overall, GATA3-positive tumors had about half the recurrence risk of GATA3-negative tumors (hazard ratio, $\mathrm{HR}=0.52, \mathrm{p}$ $=0.041)$. However, because of the strong correlation between GATA3 and ER, both of these markers should be considered together. Figure 7 shows the Kaplan-Meier plot of recurrence, by GATA3 and ER status. Because there were only two tumors that were ER negative and GATA3-positive (results not shown) the GATA3 effect could only be evaluated among ER-positive tumors. In this group, GATA3 remained somewhat protective, although the effect was attenuated $(\mathrm{HR}=0.66, \mathrm{p}=0.363)$.

The results of the final multivariable Cox model (that omitted the two GATA3positive and ER-negative tumors) are shown in Table 3. Neither GATA3 nor ER were significant, although positive expression (>20\%) of both of these markers was associated with somewhat lower risk of recurrence. In contrast, PR and Ki67 expression were both 
significantly associated with higher risk of recurrence, as was larger tumor size. GATA3 was still not significant when its expression was categorized as 0-20\%, 21-60\%, and 61$100 \%(\mathrm{p}=0.231)$, although there was some indication that a higher positive cutpoint might be more appropriate for this marker's expression (results not shown).

\subsection{GATA3 and Mortality}

A total of 51 (31\%) deaths were observed among the study's patients, with times from surgery to death ranging from 5.4 to 114.2 months (median $=36.5$ months). The patterns seen with mortality were similar to those seen with tumor recurrence but of diminished magnitude. Overall, GATA3-positive tumors showed only a small survival benefit compared to GATA3-negative tumors $(H R=0.75, p=0.310)$. Figure 8 shows the Kaplan-Meier plot of time to death, by GATA3 and ER status. When both markers were taken into account, GATA3 expression among ER positive tumors was not associated with mortality $(\mathrm{HR}=0.87, \mathrm{p}=0.728)$.

The results of the final multivariable Cox model (which omitted the two tumors that were GATA3-positive and ER-negative) are shown in Table 4. Neither GATA3 nor ER were significantly associated with survival, nor were any of the other markers. These findings did not change when GATA3 expression was categorized as 0-20\%, 21-60\%, and $61-100 \%(\mathrm{p}=0.942)$, or when the markers' degrees of expression were used as continuous predictors $(\mathrm{p}=0.766$ for GATA3). GATA3 was also not significant in analyses restricted to the first five years of follow-up after surgery $(\mathrm{p}=0.785)$.

\subsection{GATA3 and tamoxifen}

We conducted pre-specified subgroup analyses among the 121 ER-positive patients to evaluate the potential relationship between hormonal therapy and GATA3. In 
this group, 46 patients (38\%) had recorded tamoxifen treatment and the remaining 75 $(62 \%)$ did not. In multivariable analyses of recurrence, we found no significant relation between GATA3 and hormone treatment $(\mathrm{p}=0.536)$. The GATA3 protective effect appeared stronger among those who had received tamoxifen $(H R=0.57, p=0.476)$ than among those who had not $(\mathrm{HR}=1.14, \mathrm{p}=0.853)$. The same pattern was present for mortality. The relation between GATA3 and hormone treatment was not significant $(\mathrm{p}=$ 0.507), but the GATA3 protective effect was more pronounced in the tamoxifen group $(\mathrm{HR}=0.68, \mathrm{p}=0.540)$ compared to ER-positive patients not treated with tamoxifen $(\mathrm{HR}$ $=1.23, \mathrm{p}=0.743)$.

\section{Discussion}

GATA3 has been shown to be necessary for mammary gland morphogenesis, luminal cell differentiation and also for maintenance of luminal cell differentiation in mice $[8,9]$. GATA3 and ER are involved in a positive cross-regulatory loop, where each one of these factors is required for the transcription of the other gene. Furthermore, GATA3 and ER autoregulate their own expression and one would expect that GATA3 expression would be informative of the functional status of the ER pathway [10]. In welldifferentiated, low-grade carcinomas such as luminal A breast cancers both ER and GATA3 are expected to show strong expression. GATA3 has been shown to actively participate in the differentiation of luminal cells and its lack of expression is indicative of increased proliferation and the development of less differentiated tumors [16]. However, an unresolved question regarding GATA3 is its prognostic significance in patients with 
breast cancer with long follow-up information. The role of GATA3 in patients with ERpositive tumors has not been fully investigated.

The current study includes consecutive invasive breast carcinomas and correlates ER and GATA3 expression with long-term recurrence and survival follow-up. In our study, there was a consistent expression of GATA3 in ER-positive tumors (77\% of the cases). This finding is evidence that GATA3 expression is ER-dependent in most breast cancers. Furthermore, all but two of the ER-negative carcinomas were also GATA3negative, implying that GATA3 expression is dependent upon a functioning and intact ER pathway. Consequently, any predictive utility of GATA3 expression (beyond that of ER expression) essentially applies to ER-positive patients only.

Among 121 ER-positive patients, we found no significant association of GATA3 expression with either recurrence or survival, although we did confirm the importance of known pathological parameters, such as tumor size for survival and of the proliferation rate (as detected by Ki67) as a predictor of recurrence. Mehra et al studied 139 consecutive invasive breast carcinomas and measured GATA3 expression by immunohistochemistry [15]. The overall survival in that study was comparable to the experience of the patients in our study (about 75-80\% for 5-year survival, and 70\% for 10-year survival). Of the 51 deaths in our study population, 17 patients were confirmed to have died as a result of metastatic breast cancer. We were unable to verify that other 34 deaths were a direct result of the breast cancer. However, Mehra et al found a very strong and significant protective effect of GATA3 expression, with estimated 10-year survival of $55 \%$ for low GATA3 and $84 \%$ for high GATA3 (hazard ratio $=0.12, p=0.05$ ) [15]. In contrast, in our study, we estimated a much weaker and non-significant difference 
between GATA3-positive and GATA3-negative tumors (hazard ratio $=0.86, \mathrm{p}=0.73$ ). Nevertheless, given the relatively wide confidence intervals, the findings of the two studies are not inconsistent. The discrepancy may also be partly explained by differences of study populations and methods for the assessment and scoring of GATA3 expression. We note, however, that our results were not sensitive to different cut points for GATA3 positivity. When GATA3 was examined as a continuous variable and the cut-off points moved, the recurrence and survival did not change.

Since any effect of GATA3 is most likely related to tumor sensitivity to hormonal regulation, it is reasonable to expect a more pronounced effect among ER-positive patients treated with tamoxifen. In a previous pilot study of 28 ER-positive patients who had received hormone treatment, we found that GATA3-positive tumors had almost 90\% lower risk of being hormone unresponsive than GATA3-negative tumors [13]. Our current study included 121 ER-positive patients, of whom 46 had received hormone therapy. We found that the GATA3 effect was most pronounced among the latter group, with GATA3-positive tumors associated with better prognosis than GATA3-negative tumors. This finding was consistent with the results of our earlier study, although GATA3 expression was associated with only a $43 \%$ lower risk of recurrence and $32 \%$ lower risk of mortality. If this signifies a true predictive utility of GATA3, it may be a reflection of a functioning ER pathway and therefore a more predictable response to tamoxifen. Forty six of $121(38 \%)$ patients whose tumors were ER-positive were confirmed treated with tamoxifen. Presumably, the vast majority of patients with ERpositive tumors in 1995 received tamoxifen. However, we were unable to verify this information in the medical records. 
A recent study by Voduc et al looked at GATA3 expression by immunohistochemistry in breast cancer[17]. They studied 3119 cases of invasive breast carcinoma using tissue $0.6 \mathrm{~mm}$ tissue microarrays. The GATA3 antibody, like in our study was from Santa Cruz Biotechnology, but at a dilution of 1:20 (1:250 in our study). They scored GATA3 staining as negative (0) if less than $5 \%$ of nuclei stained above background, moderate (1+) if $5 \%$ to $20 \%$ of nuclei were stained, and strong (2+) if $>20 \%$ were stained. Invasive carcinomas that stained 0 were considered negative while tumors that were $1+(5-20 \%$ nuclear staining $)$ or $2+(20 \%$ nuclear staining $)$ were considered positive. Overall, our study has a slightly lower percentage of overall GATA-3 positivity (58\% vs. $68 \%$ ). Interestingly, our study had a higher rate of GATA3 positivity in ERpositive tumors (77\% vs. $39 \%$ ). Although, the GATA3 positivity was slightly differently defined from our study, Voduc et al also showed that GATA3 expression is tightly related to ER expression but lacks independent prognostic value.

Another study also showed a high correlation between ER and GATA3 using gene expression profiling and applied it to different data sets [18]. In addition, that study reported genes induced by GATA3 present in the luminal/ER-positive gene cluster. A group of tumors in this study with good outcome were better differentiated and expressed a subset of estrogen- and GATA3-related genes. Consideration should be given to patients that are ER-positive but GATA3-negative to alternative therapies instead of tamoxifen. Knowing the status of GATA3 by immunohistochemistry may help refine the optimal therapeutic approach for these patients.

In summary, in this study of consecutive patients with invasive breast cancer and long follow-up, GATA3 expression did not confer a statistically significant advantage 
with respect to recurrence or survival. However, there was some indication of a

protective GATA3 effect in the subset of patients with ER-positive tumors who were treated with tamoxifen.

\section{Disclosure/Conflict of Interest}

None 


\section{References}

1. Lowry JA, A.W., Molecular evolution of the GATA family of transcription factors: conservation within the DNA-binding domain. J Mol Evol., 2000. 50(2): p. 103-15.

2. Joulin V, B.D., Eléouet JF, Labastie MC, Chrétien S, Mattéi MG, Roméo PH., A T-cell specific TCR delta DNA binding protein is a member of the human GATA family. EMBO J., 1991. 10(7): p. 1809-16.

3. Patient RK, M.J., The GATA family (vertebrates and invertebrates). Current Opinion in Genetics \& Development, 2002. 12: p. 416-422.

4. $\quad$ Pai SY, T.M., Ho IC., GATA-3 deficiency abrogates the development and maintenance of Thelper type 2 cells. Proc Natl Acad Sci U S A., 2004. 101(7): p. 1993-8.

5. Kaufman CK, Z.P., Pasolli HA, Rendl M, Bolotin D, Lim KC, Dai X, Alegre ML, Fuchs E., GATA-3: an unexpected regulator of cell lineage determination in skin. Genes Dev, 2003. 17(17): p. 2108-22.

6. Pandolfi PP, R.M., Karis A, Leonard MW, Dzierzak E, Grosveld FG, Engel JD, Lindenbaum MH., Targeted disruption of the GATA3 gene causes severe abnormalities in the nervous system and in fetal liver haematopoiesis. Nat Genet., 1995. 11(1): p. 40-4.

7. Van Esch H, G.P., Nesbit MA, Schuffenhauer S, Lichtner P, Vanderlinden G, Harding B, Beetz R, Bilous RW, Holdaway I, Shaw NJ, Fryns JP, Van de Ven W, Thakker RV, Devriendt K., GATA3 haplo-insufficiency causes human HDR syndrome. Nature, 2000. 406(6794): p. 419-22.

8. Kouros-Mehr H, S.E., Sternlicht MD, Werb Z., GATA-3 maintains the differentiation of the luminal cell fate in the mammary gland. Cell, 2006. 127(5): p. 1041-55.

9. Asselin-Labat ML, S.K., Barker H, Thomas R, Shackleton M, Forrest NC, Hartley L, Robb L, Grosveld FG, van der Wees J, Lindeman GJ, Visvader JE., Gata-3 is an essential regulator of mammary-gland morphogenesis and luminal-cell differentiation. Nat Cell Biol., 2007. 9(2): p. 201-9.

10. Eeckhoute J, K.E., Lupien M, Krum SA, Carroll JS, Brown M., Positive cross-regulatory loop ties GATA-3 to estrogen receptor alpha expression in breast cancer. Cancer Res., 2007. 67(13): p. 6477-83.

11. Charles M. Perou, T.S., Michael B. Eisen,, et al., Molecular portraits of human breast tumours. Nature, 2000. 406(17): p. 747-752.

12. Laura J. van 't Veer, H.D., Marc J. van de Vijver, Yudong D. He, Augustinus A. M. Hart, Mao Mao, Hans L. Peterse, Karin van der Kooy, Matthew J. Marton, Anke T. Witteveen, George J. Schreiber, Ron M. Kerkhoven, Chris Roberts, Peter S. Linsley, René Bernards and Stephen H. Friend, Gene expression profiling predicts clinical outcome of breast cancer. Nature, 2002. 415: p. 530-536.

13. Parikh P, Palazzo P.J., Rose L, Daskalakis C, Weigel RJ., GATA-3 expression as a predictor of hormone response in breast cancer. J Am Coll Surg., 2005. 200(5): p. 705710.

14. Hoch RV, T.D., Baker RJ, Weigel RJ., GATA-3 is expressed in association with estrogen receptor in breast cancer. Int J Cancer., 1999. 84(2): p. 122-8.

15. Mehra R, V.S., Ding L, Shen R, Sabel MS, Ghosh D, Chinnaiyan AM, Kleer CG., Identification of GATA3 as a breast cancer prognostic marker by global gene expression meta-analysis. Cancer Res., 2005. 65(24): p. 11259-64.

16. TD., T., Luminal cells GATA have it. Nat Cell Biol., 2007. 9(2): p. 135-6.

17. Voduc, D., M. Cheang, and T. Nielsen, GATA-3 expression in breast cancer has a strong association with estrogen receptor but lacks independent prognostic value. Cancer Epidemiol Biomarkers Prev, 2008. 17(2): p. 365-73.

18. Daniel S. Oh, M.A.T., Jerry Usary, Zhiyuan Hu, Xiaping He, Cheng Fan, Junyuan Wu, Lisa A. Carey, and Charles M. Perou, Estrogen-Regulated Genes Predict Survival in 
Hormone Receptor-Positive Breast Cancers. Journal of Clinical Oncology, 2006. 24(11): p. 1665-1671. 
Figure Legends

Figure 1. Hematoxylin and eosin (400X)

Figure 2. ER positive (400X)

Figure 3. GATA3 positive (400X)

Figure 4. Hematoxylin and eosin (400X)

Figure 5. ER positive (400X)

Figure 6. GATA3 negative (400X)

Figure 7. Kaplan-Meier analysis of time to recurrence

Figure 8. Kaplan-Meier analysis of time to death

Table 1. Tumor characteristics of the entire study sample

Table 2. Treatment characteristics of the entire study sample

Table 3. Multivariable Cox regression results for time to tumor recurrence

Table 4. Multivariable Cox regression results for time to death 